\title{
Integration of Artificial Intelligence into Recruiting Young Undergraduates: the Perceptions of 20-23-Year-Old Students
}

\author{
Sara Hekkala \\ School of Business \\ Aalto University, Espoo, FIN \\ sara.hekkala@aalto.fi
}

\author{
Riitta Hekkala \\ School of Business \\ Aalto University, Espoo, FIN \\ riitta.hekkala@aalto.fi
}

\begin{abstract}
As applicants that might be subject to artificial intelligence (AI) in recruitment, students aged 20-23 years old were consulted using a qualitative approach employing focus groups. This study found that young undergraduates see AI as the future face of recruitment regardless of its challenges. Our findings are very similar to those of previous studies; however, differences arose regarding how profitable young undergraduates perceived AI and how AI should be used in recruitment. In addition, this study presents a preliminary framework for the integration of AI into recruiting young undergraduates. The framework states that AI is useful in all stages of recruiting, yet to different extents in different phases. AI is most useful in phases where grunt work is involved, and despite the integration of AI, the human touch should still be present in recruiting activities.
\end{abstract}

\section{Introduction}

HR professionals are using AI to perform tedious and repetitive tasks, the so-called grunt work [32], [33], and utilizing $\mathrm{AI}$ is a growing trend among these professionals [31], [32]. A recent study [31] argued that $\mathrm{AI}$ is making its way into becoming one of the cornerstones of the recruitment industry. However, researchers are not unanimous on how much AI is already applied into recruitment processes: Leong [18] saw potential for AI in the near future, as opposed to others who already claimed to see AI's impact on recruitment [31], [32]. Furthermore, when looking at issues related to recruitment, it is not enough to analyze issues only from the recruiter's perspective [4] Van Esch et al. [32] believed that understanding applicants' attitudes toward recruiting with AI would help integrate AI into recruiting activities seamlessly. Moreover, it is vital to understand the differences between the mindsets of digital natives and the already existing workforce [10].
Parnas's [22] take on intelligent machines is that they should be created for the purpose of substituting those areas of life where people do not excel. For example, human limitations and biases are reduced when recruiting using AI than without [5], [29]. Because AI-powered programs eliminate prejudices, they could be considered unbiased [31]. However, AI programs are also self-learning and therefore prone to learning biases [28], [33]. Additionally, these programs run on the data that they are programmed with, even if that is biased data [13]. This is why it is important to research the usage of AI in recruitment and to understand how the machines work to avoid contradicting what AI is ideal for-unbiased recruiting. The rumor surrounding $\mathrm{AI}$ is that it is here to replace people in the workplace while leaving them unemployed [9]. However, [19] argues the opposite: the more organizations invest in technologies, the more they have time and assets to invest in the people of the workplace. To dispose of these negative attitudes (e.g., AI replacing jobs) and to enable the seamless integration of $\mathrm{AI}$ into the workplace, organizations need to understand what $\mathrm{AI}$ is and what it is not [13].

A lot of current research focuses on the benefits of using AI in recruitment, but hardly any studies investigate, for example, how digital natives view this subject. It is important to understand how the dynamics of the workplace have begun to change with input from the younger generation of digital natives [10]. To begin addressing the research gap, this study focuses on a small sliver of digital natives in a Nordic country by investigating how young undergraduates (the digital natives) perceive the use of $\mathrm{AI}$ in recruitment.

This study is guided by the following questions: 1 . what is the perception of young undergraduates toward the use of AI in recruitment and 2. to what extent do young undergraduates believe that AI can be utilized in recruitment? In terms of theoretical contribution, we provide initial information on understanding how young undergraduates perceive the successful usage of $\mathrm{AI}$ in recruitment. This is illustrated with a framework based on our findings. 
The rest of this paper is organized as follows. In the next section, we present the basic ideas related to recruitment as a part of $\mathrm{HR}$. We also outline the benefits and challenges of $\mathrm{AI}$ in recruitment. The following three sections describe the research case, the research method, and our findings. In the final sections, we discuss our findings and conclude the paper.

\section{Theoretical Background}

This literature review focuses on different aspects of recruitment and what has been written about using AI in recruitment from the individual's viewpoint. A study by [18] found that recruiters can spend as long as eight and a half hours manually studying 100 candidates that have applied for one specific job. This feels like an unreasonable number of hours put into filling one vacancy, especially when it is claimed by [5] that up to $80 \%$ of the applicants for each job are unsuitable. Pitt [23] advised recruiters to perform thorough background checks on applicants. Thus, it is safe to argue that the traditional assessment method is slow and ineffective [12], as putting excessive hours into recruitment does not necessarily equate to a favorable outcome [8]. Inevitably, this raises the question of how many hours are actually spent studying resumes; some vacancies have hundreds of applicants, and some processes have multiple recruiters screening the same applications. To escape the grunt work of recruitment, some organizations may resort to shortcuts [18], such as internal hiring, which is one of the pitfalls of conventional recruiting [11]. Hence, companies often resort to employee referrals in their recruiting activities [11], which could result in claims of nepotism.

\subsection{Artificial Intelligence in Recruitment}

McCarthy [21] defined AI as "the science and engineering of making intelligent machines, especially intelligent computer programs. It is related to the similar task of using computers to understand human intelligence, but AI does not have to confine itself to methods that are biologically observable." AI-driven programs can create algorithms, observe patterns, and combine data by themselves [9], [29]. They have the ability to learn from themselves without being programmed to do so and thus gradually become smarter and more efficient [19], [29], [33]. Due to the AI programs' abilities to reflect on their input, AI programs are often described as machines that possess human-like intelligence [19], [21].

For the purposes of this research, AI will be defined as any computer program and system, software, or machine that can be described as intelligent, smart, self-learning, or self-correcting. Essentially, this means machines, programs, or applications that can operate and develop on their own and without human intervention.

There is general reluctance from HR professionals when it comes to utilizing AI [3]. Some workers are afraid of AI taking over their jobs [33]. This may be because many perceive AI as advanced, even dystopic robots that are taking over the workplace [19]. Technology has reformed processes and forced companies to adapt their strategies to technological advancements [1]. However, the literature is not unanimous on to what extent companies have already begun to utilize AI. Leong [18] argued that AI is an already integrated part of the workplace, whereas Benfield [5] referred to AI as "an emerging technology in HR." Nonetheless, there is general consensus around the fact that AI can be useful in the workplace. If HR professionals learn to use AI effectively, the possibilities for analyzing and keeping track of current employees [29] and for developing innovations and the skills needed for it are endless [13], [25]. In addition, using $\mathrm{AI}$ in $\mathrm{HR}$ functions reduces the time spent on routine jobs, such as applicant screening and tracking [18], [25].

Despite all the opportunities that the literature points out, there are some problematic features of AI. For example, Scherer [29] went as far as stating that using AI in HR has implications on civil rights and stated that AI systems are prone to be biased because even if the AI program itself is objective [31], the data that has been put into the system will be subjective [29]. It was also argued by [31] that although AI systems can be programmed to avoid bias in their decision making, they learn from patterns, which can be biased [33]. It is important to realize that the program will be only as good as the data that is put into it and the mechanism that runs it [9], [13], [29], [33]. Scherer [29] stated that applying AI into HR is a matter of training HR professionals to use the machines right [13], [29]. By thinking critically and recognizing the limitations that technology has, AI can be useful in HR, although it will not fully replace the human aspect for some time [29]. Disappointingly, the literature limits itself to this idea and does not present advice on how these professionals should be trained or how to use AI in HR in a beneficial way.

Within the HR sector, AI tools have been built mostly for recruitment and hiring activities [3]. Within these activities, AI is applicable primarily in the first steps of the recruitment process, such as sourcing and screening [32]. In recent years, the interest in using AI in recruitment has grown not only among large corporations but among smaller companies as well [3], 
[32]. Despite being a trend, the companies actually utilizing AI in their HR are the vast minority [13], [25], [31]. The reason for this may be the negative aspects of AI. According to Baraniuk [3], systems (e.g. applicant tracking systems [ATSs]) that use $\mathrm{AI}$ in screening applicant $\mathrm{CVs}$ are unfair to those with nontraditional CVs. The challenge is making CVs that please both the ATS and human reader [28]. An ATS uses keywords regarding capabilities, education, or previous employment that are preselected by the recruiter [28]. If an applicant does not have the right keywords in their resume, the $\mathrm{CV}$ might never make it through the screening phase to be read by a human [3].

Zielinski [33] considers inefficient data in AI programs to be the biggest problem in recruiting with AI. For example, there are programs that utilize AI in the screening process by picking out the best $\mathrm{CVs}$ among the many applicants [18]. However, according to [8], people tend to exaggerate their positive attributes on their CVs. Naturally, this could result in the machine picking out applicants that might not be competent for the job after all. This enhances the point made above about AI being only as good as the input data, which may be a reason why many authors highlight that the human aspect should not be forgotten or underestimated [9], [27], [28], [29], [33]. According to an extensive survey by [19], recruiting and hiring professionals do not see AI as something that can replace the phases of recruitment involving peoplebuilding relationships, interviewing, and phases where emotional intelligence is required. The study by [13] abides by this idea and claims that machines are mainly good for repetitive, simpler tasks.

\subsection{Benefits of $A I$ in Recruitment}

Regardless of the conflicts that the literature brings up about the usage of AI, the benefits of AI are overexceedingly more apparent. The survey by [19] found that close to $80 \%$ of recruitment professionals believe that AI will have a somewhat significant effect on recruiting and that using AI will be most helpful in sourcing, screening, and nurturing candidates. Furthermore, most recruiting professionals agree that using AI in recruitment will be time-saving [5], [12], [13], [19], [31]. Martin [20] argued that using smart technologies in recruiting can save monetary assets. If time is eliminated from assessing irrelevant resumes [18], hiring professionals will have more time for the truly suitable applicants and more assets for implementing strategic recruiting [5]. It was also stated by [31] that using AI will change the currently dominant strategies for recruiting. Eventually, by using AI properly, hiring professionals will grasp a better view of how the programs can be used effectively and without human bias to ensure the most favorable outcomes [29].

Based on the literature, the main benefits of using AI in recruitment are how quickly and effortlessly AI can process, attain, and reorganize data compared to humans [31]. AI systems can go from as far as evaluating candidates' honesty and emotional intelligence just by analyzing video interviews [33] to assessing the candidates' personalities through their online and social media presences [28]. The literature seems to almost exaggerate the benefits of using AI in recruitment; in fact, Tolan [30] reminds us that AI fails to live up to the expectations of HR professionals. It has been highlighted that AI will not replace the recruiter, but it will rather assist in the process of recruiting [5]. Some actual applications of AI in recruitment that authors have brought up are social media websites [3], [11], [12], [15], [17], [20], [28], [29], [31], [33]. LinkedIn is the most referenced website among the literature [3], [11], [15], [20]. Social media platforms are ideal for the efficient communication between the applicant and recruiter [11], [17] and represent remarkable databases of information about applicants [11], [12], [28], [33] and even about the hiring company [2], [29], [31]. It may be even useful for the employee to have a website dedicated to recruiting [20].

\subsection{AI in Recruitment from the Applicant's Perspective - Benefits and Challenges}

Even though researchers recognize the effect that AI has on recruitment today, the subject has not been studied much from the individual's perspective [32]. It is critical to view the transformation of the recruitment processes from the applicant's point of view in order to fully understand this new era of recruitment [4]. Even more so, the future of the workforce is a generation that is accustomed to advanced technology and doing things online; therefore, it is essential for recruiters to be aware of this to be able to recruit the best applicants [17]. By using AI, applicants can receive real-time, unbiased feedback when applying for jobs [18]. However, the promise of unbiased AI programs should be assessed critically: they are self-learning and therefore prone to learning prejudicial patterns [29]. Van Esch et al. [32] found that an applicant's motivation to use technological devices has a positive effect on how willing the applicant is to apply for a job that uses AI in their recruitment. Essentially, Van Esch et al. [32] established that the applicant's attitudes toward AI affect the recruitment process. The study also found that other factors affecting an applicant's willingness to apply for a job are attitudes toward the organization and the applicant's levels of anxiety with 
using AI. However, it is not clear whether companies should be completely transparent about their AI-based recruiting activities. Should the usage of AI be mentioned, for example, in the job posting?

On another note, as mentioned above, social media and utilizing AI in that way are effective panels for interaction between the recruiter and applicant. Adequate communication between both parties is important and perceived as useful [30]. Therefore, adding $\mathrm{AI}$ in the communication between the hiring staff and candidates can be profitable. Tools that can be used for this are AI-powered interactive chatbots on the companies' webpages or social media sites [31] that answer questions for the candidates and even provide feedback in real time [18], [19]. Chatbots that operate around the clock are useful, especially when communication happens across several time zones [13]. Because the integration of AI into recruitment is not widely recognized by applicants, they may miss out on potential job offers. Edwards [11] claimed that many people applying for jobs do not have a LinkedIn profile or fail to update it, which results in missed opportunities, as LinkedIn is one of the most used websites by recruiters. Furthermore, Faliagka et al. [12] presented an e-recruitment system that assessed candidates' personalities and their fit for a position by analyzing their social media presence. If organizations applied systems like these into their recruitment, an active social media presence would be required of applicants to guarantee a diverse image of them [29].

However, it has been argued even that using AI in HR will result in the violation of the applicant's civil rights [29]. Scherer [29] made a valid point: people often have private online profiles, which are private for a reason. Profiles that have little or dated information are not trustworthy presentations of the applicants' personalities. In addition, if applicants feel that AI devices are invading their privacy, according to [32], these negative feelings will affect the applicants' overall motivation to apply for jobs. In addition, it would seem over-excessive to require applicants to have a social media presence at all — especially digital immigrants, who are not as accustomed to technical devices as digital natives are.

A plausible explanation for the careful integration of AI into recruitment can be the generational differences between digital natives and digital immigrants. The majority of senior managers in organizations today are digital immigrants, and the generation entering the workforce are digital natives [10]. Prensky [24] claimed that there is a digital language barrier between digital natives and digital immigrants. It was even argued by [10] that the clash of the two generations will affect the way business is conducted. The ways digital natives and digital immigrants process information, communicate, and are accustomed to doing things are considerably different, which can result in disputes between the two generations. Prensky [24] argued that in order to overcome the language barrier, it should be the digital immigrants who need to make an effort because it is very unlikely that the new generation would be willing to take steps back [13].

\section{Research Methods and Design}

Because young undergraduates (an essential part of digital natives) are the future of the workforce, it is important to understand not only their attitudes, but also how they process information compared to digital immigrants [10]. The following section describes the sampling, data collection processes, and focus groups of this study.

\subsection{Data Collection and Focus Groups}

The participants for the focus groups were recruited based on a few defining characteristics. First, each participant had to be a young undergraduate (someone born between 1980 and 1999) and was required to have experience in applying for jobs. The participants were gathered by convenience sampling, which means that everyone was an undergraduate majoring in International Business at the same university in the same Nordic country. Their educational background might make them more aware of things related to conducting business, such as HR activities and recruiting. The focus groups were semi-structured and had a set of standard questions, but defining questions were asked to clarify points. The focus groups were recorded electronically. A focus group suits the purposes of this research because the objective is to explore the underlying perceptions young undergraduates have on recruiting with AI [26]. Focus groups can offer insights into the group dynamics and therefore enable the researcher to form a social understanding of the concept being studied [26]. For this study, it seemed more suitable to have smaller groups than the maximum of ten people suggested by Krueger [16] to encourage more input from each individual. The focus groups were conducted in person between three participants and the interviewer. Additionally, as proposed by [16], the focus groups were repeated three times with different people to ensure that the findings could be contrasted between different groups. Therefore, the total number of participants was nine. The details of the participants are summarized in Table 1. 
Table 1. The details of the focus group participants

\begin{tabular}{|l|l|l|l|}
\hline Participant & Focus group & Age & Gender \\
\hline 1 & 1 & 22 & Female \\
\hline 2 & 1 & 21 & Male \\
\hline 3 & 1 & 21 & Male \\
\hline 4 & 2 & 21 & Female \\
\hline 5 & 2 & 22 & Male \\
\hline 6 & 2 & 23 & Male \\
\hline 7 & 3 & 21 & Female \\
\hline 8 & 3 & 20 & Male \\
\hline 9 & 3 & 21 & Male \\
\hline
\end{tabular}

Additional recommendations [16] were used in the focus groups, such as requesting the participants to sit in a circle and asking open-ended questions. The interviewer first told the participants why they had been selected and then defined what AI is. Then, the participants were asked about their initial thoughts on recruiting with AI. Next, they were shown a YouTube video by Canadian HR Reporter [7] where two professionals who were very familiar with the usage of AI talked about how AI is profitable in recruitment and how it is actually used. After watching the video, the focus groups were asked more questions about the usage of AI in recruitment and whether they had participated in an AI-based recruitment process in applying for jobs. Furthermore, the participants were also inquired about their presence on LinkedIn, the most used social media website by recruiters, as shown by the literature [11].

\subsection{Thematic Analysis}

Thematic analysis is one of the key methods for analyzing qualitative data [6]. It is widely applicable and flexible, and since there are no right answers for interpreting data, it provides the analyst with the theoretical freedom to discover patterns. Focus groups are most often analyzed with thematic analysis [6], [14]. Even though there is no clear-cut theory for this method, Braun and Clarke [6] created six phases for conducting thematic analysis, all of which were followed in this study: 1) familiarizing yourself with your data, 2) generating the initial codes, 3) searching for themes, 4) reviewing the themes, 5) defining and naming the themes, and 6) producing the report. The data analysis for this study began with transcribing the focus groups as soon as possible, as suggested by Krueger [16] in his framework. Following this, the full transcripts were read through repeatedly while simultaneously generating initial ideas about similarities among the focus groups. The transcripts were subsequently coded physically according to what may be interesting or useful for the research objectives, focusing on the manifest content. After the initial coding, the codes were assessed to look for similarities, which would later become the themes. Five dominant themes were identified: efficiency, impartiality, conformity, human interaction, and uncertainty.

\section{Findings and Analysis}

This section discusses the themes and gives more insights into how the themes emerged in the focus groups. We will also analyze the themes in relation to the codes and transcript material.

\subsection{Theme 1: Efficiency}

One of the first themes that stood out from the transcripts was efficiency. In this context, efficiency means making the recruitment process more streamlined and cost-effective by utilizing AI. The participants recognized the effectiveness of using AI in recruitment from both the organizational and individual perspectives. However, some participants felt that even though AI makes the recruitment process faster and cost-effective, it would still cost the organization money to hire people to program and monitor the AI systems: "If you have certain requirements and the AI just goes through everything and finds what you need, that is not exactly a perfect way of doing things, but it's still much faster, much more efficient" (Participant \#5).

The time-saving aspect of efficiency was realized from the applicant's perspective as well. While the organizational perspective on time saving was recognized by all focus groups, the individual perspective was evident in only one: "It feels so frustrating...I won't get any replies for like a month ... so the AI could help with this" (Participant \#9). Recognizing the efficiency of using AI in recruitment means that young undergraduates are aware of the potential benefits of using AI. However, the focus groups talked about the time-saving aspect with regard to job posts that have a notable number of applicants, which would suggest that they see AI as being useful for larger companies. One focus group pointed out that AI does not add value to the recruitment process if there are only a handful of applicants for a position.

\subsection{Theme 2: Impartiality}

The impartiality theme attempts to describe how the focus groups recognized the importance of 
impartial recruiting systems. The video that they were shown talked about biases and their negative effects on minorities applying for jobs. The focus groups realized the benefits of AI in that it is theoretically unbiased; in other words, AI-powered programs may offer applicants equal chances of getting interviews. Nonetheless, all three focus groups were skeptical about the elimination of biases. The participants realized that even if AI programs are able to recruit without bias toward demographics, those biases would still be present in the recruiters themselves and might therefore build the systems to replicate their biases. Biased employers and AI programs were generally deemed unacceptable:

"It's nearly impossible to delete all of the biases through AI, but I find it might delete some. But it does require the person, who, for example, sets the parameters to consciously understand the biases that they have" (Participant \#4)

"I think one point to consider is that if your employer has a bias towards you, then well, at least from my perspective, I wouldn't necessarily want to work for the people” (Participant \#8). However, one focus group noted that bias in recruiting is not necessarily a bad thing: "It could be fitting to your company culture to have certain attributes and such. So, being biased towards that kind of thinking, for example, if you are a very strict hierarchy, you definitely want people who can actually follow orders and such" (Participant \#5)

Despite the notion that bias may not be a bad thing, the focus groups were unanimous about the benefits of removing biases toward people's names, backgrounds, and personal lives. Therefore, using AI to understand and remove demographic biases from the recruiting process is beneficial and should be applied.

\subsection{Theme 3: Conformity}

Conformity as a theme in the focus groups had a slightly negative connotation. The impartiality theme overlaps with some of the features of conformity. Here, impartiality is wanting equal treatment for all applicants regarding their demographics; on the other hand, conformity is not wanting to be treated like everyone else. Essentially, conformity here means that through using AI programs, the participants felt that aspects of their personality were lost due to generalizations. The participants believed that they were not able to express themselves with these generic words that they are supposedly required to have in their applications: "A general opinion about the AI is that it sucks that when you're filling up your $C V$ you have to use generic terms and you can't put your personality in the CV, right?" (Participant \#2). Conformity was evident especially when the participants were talking about programs that pick out the best applicants by skimming through CVs searching for the right keywords. It was interesting to see that all focus groups listed keywords that the programs were looking forthat is, those generic keywords that they should be using: "Good at problem solving, and the most generic: my worst quality is that I'm a perfectionist" (Participant \#3); "Everybody needs to be innovative and creative and energetic ... Everybody has to be entrepreneurial" (Participant \#5); "If you're looking for self-initiative, active, anything, then you're probably going to put those qualities in your application if you have them" (Participant \#7).

This would suggest that the participants want to be recognized for their differences and personal attributes. The respondents also stated that if they knew that a certain job application process would be using AI to recruit, their behavior in the application process would change (e.g., using the generic keywords that will be picked up instead of their personalized CVs). This may also be why all focus groups stated that they want transparency from companies on whether they are using AI in recruitment or not. This indicates that the informants believe that a person is more competent to judge an applicant's personality than a machine.

\subsection{Theme 4: Human Interaction}

Despite the benefits that the informants saw in using AI in recruitment, it was clear that maintaining the human touch was important. The desire to have people present in the recruitment process can be due to either a general mistrust in machines or because the participants felt that machines are not capable of judging certain features as well as people. For example, the informants pointed out that machines cannot tell how effective people are at communicating in the workplace or what a person is like. This is also evident in the conformity theme. The applicants need to blend in to succeed in an application process with AI: "Can you, you know, get the same level of interaction between the worker and the employee in the hiring process [when using AII?" (Participant \#3).; "In essence like, how do you appear... what kind of image you leave behind when you talk to someone, and I think machines cannot interpret that" (Participant \#9).

The human interaction as a theme was also evident in how much importance the focus groups placed on the interview section of the recruiting process, presumably because that is the place where human interaction is most present. All focus groups stated that using AI in recruitment is acceptable as long as they get interviewed. Undoubtedly, interviews are essential for the recruiter in determining the personality of the 
applicant, which seems to be important for the young undergraduates as well: "I think the key is to try to get the interview" (Participant \#6). This was emphasized by the focus groups while discussing the importance of receiving feedback from the process and developing as a person: "If there's no level of feedback, you send in your application and it doesn't make it to the actual person, you have no idea what went wrong" (Participant \#5).

\subsection{Theme 5: Uncertainty}

The final recurring theme in the focus groups was uncertainty toward several aspects of recruiting with AI. For example, the participants seemed unsure of how AI programs actually work and to what extent they can be used. In addition, as mentioned above, the participants felt that they had to change how they behaved in the recruiting process when AI was involved-perhaps because they were not sure what the AI programs were looking for. Uncertainties were also present in how profitable the participants thought AI programs are. Furthermore, several respondents felt that AI is still a developing technology and still in its primitive phase. Due to the underdevelopment of AI, the focus groups felt it is not profitable in the recruitment process in its current form. However, this could be because the participants seemed to be unaware of the full capabilities of AI. They reported that they were concerned, skeptic, and intimidated by AI-powered programs: "Very pessimistic, like I would like, think that I probably wouldn't get an interview or something, I don't know why but I'm skeptic..." (Participant \#1).

"And now, if the AI is just on the level that look for these keywords on the application form, it doesn't really change the method just to medium. That it just happens on a different platform" (Participant \#5).

"I'm not sure if I understood the face recognition thingy correctly, but it seemed a bit intimidating to me ... something is going to scan your face and see, like, your deeper soul" (Participant \#7).

There also seemed to be general uncertainty among the applicants as to what is good behavior or a good job application for a company that uses AI in recruitment. This was also looked at from the organizational perspective: what if the recruiter fails to recognize important features that the applicants need? The participants as applicants would want to know the parameters that recruiters set for the open positions: "If the recruiter themselves does not identify certain keywords that are synonyms, or antonyms, or whatever ... it can actually damage the recruitment process quite a bit" (Participant \#4); "There was this advice for applying for jobs now that there is AI: always copy the texts from the recruitment advertisement, or whatever, make it white, and add it to your CV so the computer [snaps fingers] picks it up" (Participant \#2).

\section{Discussion}

The five themes identified in Section 4 will be discussed separately in the light of the literature and further assessed. We also present a framework.

\subsection{Assessment of Theme 1 (Efficiency)}

The efficiency theme was evident in both the focus groups and the literature. Efficiency as a theme encompasses the view that AI would make the recruitment process more streamlined and costeffective. The focus groups recognized the time-saving and money-saving aspects of AI in recruitment. According to the literature, the conventional ways of recruiting are very time-consuming [12], [18], and AI is useful in reducing the unnecessary hours spent on the recruiting processes [12], [13], [19], [25]. The reason for calling this theme "efficiency" is that despite the reduced amount of time spent on recruiting, the quality of the recruitment process will be unaffected [31]. In addition, as time is taken away from tedious tasks, HR professionals have more assets for other jobs, such as strategic recruiting [5] or concentrating on their employees and building their strengths [18], [19]. Furthermore, utilizing AI in recruitment saves monetary assets [20]. However, cutting down on time and money may be sequential events. As the hours spent on recruiting are mitigated, the amount of resources put into recruiting is reduced. Nevertheless, it should be noted that the focus groups recognized the money-saving aspect more than the literature did. All in all, the findings would indicate that HR professionals and informants have similar views on the efficiency of utilizing $\mathrm{AI}$ in recruitment.

\subsection{Assessment of Theme 2 (Impartiality)}

Impartiality was another theme that emerged from the focus groups and is supported by claims in the literature that AI-powered programs may eliminate bias in the recruitment process [5], [7], [29], [31]. This was an important topic for the young undergraduates; the focus groups realized that eliminating biases was a good thing, but doing so only in the screening phase of recruitment is not enough. The groups, as well as the literature, additionally pointed out that AI-powered programs are generated by people and are therefore prone to err. AI machines are only as good as the programs that run it [9], [13], [28], [29], [33]. 
However, an issue that was brought up by the focus groups was that having biases may not necessarily be bad. It may be fitting to have recruiting systems biased to select from the pool of applicants those personalities that suit the hiring organization. This was not addressed in the literature - the word "bias" had a negative connotation throughout the reviewed articles and was usually deemed a harmful thing. Nevertheless, general impartiality in using AI appears to be an important factor for young undergraduates.

\subsection{Assessment of Theme 3 (Conformity)}

One theme found in the focus groups that is not as apparent in the literature is conformity. Here, conformity means that through using AI programs, the young undergraduates felt that aspects of their personalities were lost due to generalizations, such as the mandatory inclusion of generic keywords in their resumes. Based on the efficiency theme, the participants' need to express their personalities, and the point about biases not being all-bad, it can be deduced that it is important for young undergraduates that their personalities fit the organizations they work for. The focus groups pointed out that people who fail to include the right keywords in their resumes may not be picked up by the ATS [3], [28]. Additionally, there are programs for interpreting an applicant's personality based on their social media presence [12], [28] or by analyzing video interviews [33]. The focus groups were doubtful of machines being able to judge character. Furthermore, as stated in the findings section, the focus groups felt that they would appreciate transparency from the recruiter if they were utilizing AI in recruitment. This is supported by the findings in [32], where applicants' favorable attitudes toward AI and successful recruiting with AI correlate positively. Young undergraduates seem to be more pro-AI in general than the literature and thus may have positive attitudes toward utilizing AI in recruitment.

\subsection{Assessment of Theme 4 (Human Interaction)}

Human interaction as a theme is something both young undergraduates and the literature [9], [27], [28], [29], [30], [33] argue is important, almost vital, in recruiting. As mentioned above, young undergraduates do not believe that machines are capable of the same type of interaction as people are, and the literature agrees with this. Tasks that require interactive skills or emotional intelligence, such as interviewing and engaging applicants, are not tasks that should be done by AI [13], [19]. However, the focus groups relate the issue of human interaction to the interview part of the recruitment process and to being assessed by a human being, which is not something that the literature discusses. Extant studies approach the concept of human interaction from a different perspective: machines cannot replace complex tasks - which are not repetitive or simple in the way that, for example, scanning keywords is. The young undergraduates, on the other hand, pointed out the human features that AI cannot replace, such as the judging of character, interaction, or an interview. The participants identified the harm that may be caused to applicants when human interaction is not present.

\subsection{Assessment of Theme 5 (Uncertainty)}

What is present in much of the discussion above is the theme of uncertainty. In this context, uncertainty is the combination of underlying unfavorable feelingssuch as mistrust, skepticism, and concern - toward AI in recruitment. For example, the focus groups displayed uncertainty in connection to how AIpowered programs work, how applicants should behave when AI is used, and the profitability and overall applicability of AI. Many of these causes of uncertainty are justifiable, as they are the same causes that the literature points out. First, when new technologies are introduced into the workplace, employees are usually unwilling at first to integrate the technologies into their work [3], [5], [25]. Second, the literature does not provide instructions for applicants on how to apply for jobs when AI-powered systems are in charge of, for example, screening. Third, AI programs are limited and not yet widely accepted by employees, perhaps due to concerns about losing their jobs to AI [9], [19], [33]. Finally, the overall applicability of AI is controversial within the literature as well, as some texts believe that AI-powered machines are not suitable tools for the workplace in their current form [3], [22], [28], [30], whereas others are convinced of their profitability [13], [18], [19], [25], [29], [31], [33].

\subsection{Further Themes Identified}

In the study by [19], it was found that almost $80 \%$ of recruitment professionals believe that AI will have a somewhat significant effect on recruiting, whereas all the young undergraduates in the focus groups thought that AI will be the future face of recruiting. However, the participants perceived AI as potentially useful for recruiting activities that have to deal with a large number of applicants, an idea not specified in the study by [19]. Furthermore, it was found that the vast majority of applicants who are young undergraduates are not active on LinkedIn, whereas the literature found 
that LinkedIn is the prevalent social media website used by recruiters [3], [11], [15], [20].

\subsection{Development of Framework}

This section combines the perceptions from the focus groups and literature to develop a framework on utilizing AI in recruitment from an organizational and from young undergraduates' perspective. The new framework is illustrated in Figure 1.

\section{Figure 1: Framework for the integration of Al into recruiting young undergraduates}

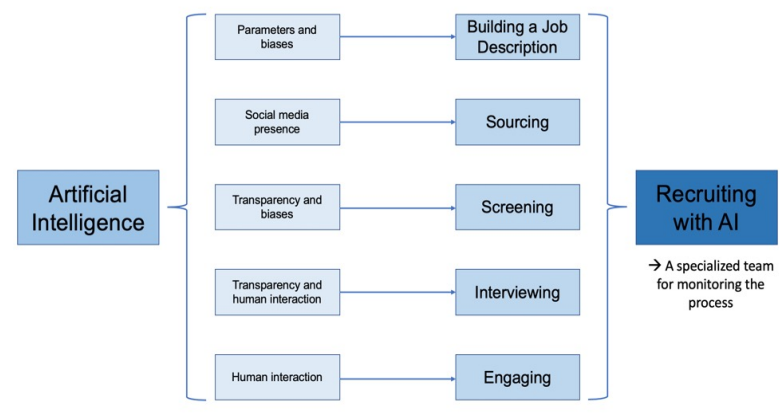

In the framework, the phases of recruitment are building a job description, sourcing, screening, interviewing, and engaging, and the utilization of AI was devised around them. The framework attempts to offer guidelines as to how AI should be used in each of the stages. In addition, under "Recruiting with AI," the framework takes into account that this process should be conducted and monitored by a specialized team that understands recruitment and especially AI. First, in the building a job description stage, AI can be utilized in finding the right keywords, parameters, and standards that could be extracted from, for example, previous hiring data within the organization. However, in this stage, the people monitoring the AI programs that go through the old data should be actively aware of all the possible biases - both positive and negative - that may emerge. Demographic biases should be mitigated in this stage. The parameters should be set so that the applicants do not have to alter their behavior when applying for jobs that use $\mathrm{AI}$ in recruiting: there should be room for personalization. In the second section, in sourcing applicants, the literature advises recruiters to build a social media presence. This may be useful, especially because young undergraduates are accustomed to working with technologies, but it should be pointed out that young undergraduates do not seem to be present on the traditional recruiting medias, such as LinkedIn. Therefore, utilizing AI in the sourcing stage should be attempted by other types of social media presence. For example, a useful AI tool in this phase would be a chatbot that answers the applicants' questions. The next phase after sourcing is screening. The young undergraduates found this step of the recruitment process to be the most negatively associated with the use of AI. However, this is the stage of recruitment where AI is most useful, especially for those job postings that have hundreds or more applicants. The recruiter should be transparent in this phase about their parameters and requirements for the open post, as in the job description phase. This section should also try to provide the unsuitable applicants feedback about why they were not chosen. Using AI in generating the feedback may be useful and more time-effective than replying individually to each applicant. Using AI in the screening process should also eliminate possible demographic biases.

The young undergraduates as well as the literature noted that it was important to have human interaction present in the final stages. If the recruiter does initial video interviews and uses AI to interpret them, the organizations should be transparent about this. The participants claimed that it was important that their personalities were judged by a human. The engaging phase of recruitment should also maintain its human interaction, as it consists in introducing the candidate to their new position. Finally, it should be stated that young undergraduates and people in general are still too hesitant about AI to allow for the complete digitalization of recruitment.

\section{Limitations and Conclusion}

This study presents a number of limitations. First, the framework cannot be generalized, as it is based on only a very small sample of students at one small institution in a Nordic country. In addition, no HR professionals were directly involved in the research. We should also note that young undergraduates and digital natives from, for example, a different continent, may have different perceptions on the topic. Furthermore, it was not clear whether the participants had actually experienced AI in recruitment or they were informed mostly by the discussion and the shown video. Therefore, their perceptions may have been different if they had factually experienced AI in practice. In addition, the framework attempts to guide recruiters, but the literature on which the framework is based is not specifically on recruitment in the Nordics. It is also worth mentioning that the framework may change with input from HR professionals - a surely interesting subject for future research. It is evident from the literature and the focus groups that applying AI in recruiting is profitable for positions with many applicants. Therefore, the utilization of AI in 
recruitment remains the concern of larger organizations, at least for now.

\section{Acknowledgments}

We would like to warmly thank Associate Professor Kate Black for her very valuable comments and thoughts on the earlier versions of this research.

\section{References}

[1] A. Apatean, E. Szakacs, and M. Tilca, "Machine-learning based application for staff recruiting," Act. Tech. Nap., vol. 58, no. 4, pp. 16-21, Dec. 2017.

[2] G. C. Banks et al., "Strategic recruitment across borders: An investigation of multinational enterprises," J. Manage., vol. 45 , no. 2 , pp. 476-509, Aug. 2018

[3] C. Baraniuk, "The AI headhunters," New Sci., vol. 228, no. 3045 , pp. 20-21, Oct. 2015.

[4] A. E. Barber, Recruiting employees: Individual and organizational perspectives. Thousand Oaks: Sage Publications, Inc., 1998.

[5] S. Benfield. "How artificial intelligence is improving talent acquisition." HR.com.

https://www.hr.com/en/magazines/all articles/how-artificialintelligence-is-improving-talent-ac j0c8jqu4.html (accessed Sep. 28, 2020)

[6] V. Braun, and V. Clarke, "Using thematic analysis in psychology." Qual. Res. Psychol., vol. 3, no. 2, pp. 77-101, Jul. 2008 [Online]. Available:

https://www.researchgate.net/publication/235356393_Using Thematic_Analysis_in_Psychology

[7] Canadian HR Reporter. Using AI for Recruitment (Jun. 5, 2018). Accessed: Sep. 28, 2020 [Online Video]. Available: https://www.youtube.com/watch?v=MwBLutG-ZOo

[8] S. Dalgleish, "Recruiting quality," Quality, vol. 44, no. 6, p. 14, Jun. 2005.

[9] M. J. Dennis, "Artificial intelligence and recruitment, admission, progression, and retention," Enroll. Manage. Rep., vol. 22, no. 9, pp. 1-3, Nov. 2018.

[10] D. Dumeresque, "The next generation: Its impact on the business landscape," Strateg. Dir., vol. 28, no. 9, Aug. 2012.

[11] D. C. Edwards, "People and technology: A winning recruiting combination," Career Planning \& Adult Develop. J., vol. 32, no. 3, pp. 45-50. 2016.

[12] E. Faliagka, M. Rigou, and S. Sirmakessis, S., "An erecruitment system exploiting candidates' social presence,' Lect. Notes. Comput. Sci., pp. 153-162, Sep. 2015.

[13] RES Forum, "Shiny New World? Global Mobility in The Age of Artificial Intelligence and Robotic Process Automation," 2019. [Online]. Available:

https://theresforum.com/newsletter/res-forum-weekly-digesttuesday-19th-march-2019/

[14] H. Joffe, "Thematic analysis," in Qualitative Research Methods in Mental Health and Psychotherapy: A Guide for Students and Practitioners, $1^{\text {st }}$ ed. Chichester, UK: John Wiley \& Sons, 2011, ch. 15, pp. 209-203 [Online].
Available:https://www.researchgate.net/publication/2300005 72_Thematic_Analysis. Accessed: Sep. 28, 2020.

[15] E. Kronz, "Acquiring and developing leaders on a global or multinational scale," Strategic HR Rev., vol. 13, no. 6, pp. 249-254, Oct. 2014.

[16] R. A. Krueger. (2002). Designing and conducting focus group interviews [Online]. Available: https://www.eiu.edu/ihec/Krueger-FocusGroupInterviews.pdf [17] N. Kunes, "The advantage of technology in recruiting property management talent," J. Prop. Manage., vol. 83, no. 4, p. 33, Jul./Aug. 2018.

[18] C. Leong, "Technology \& recruiting 101: how it works and where it's going," Strategic HR Rev., vol. 17, no. 1, pp. 50-52, Feb. 2018.

[19] LinkedIn Talent Solutions. "Artificial intelligence: Your secret workhorse," LinkedIn.com.

https://business.linkedin.com/talent-solutions/recruitingtips/artificial-intelligence-your-secret-workhorse (accessed Sep. 28, 2020).

[20] L. Martin. "What is recruitment? Definition, recruitment process, best practices," cleverism.com.

https://www.cleverism.com/what-is-recruitment

[21] J. McCarthy. (2007). What is artificial intelligence? [Online].

Available:

http://jmc.stanford.edu/articles/whatisai/whatisai.pdf

(accessed Sep. 28, 2020).

[22] D. L. Parnas, "The real risks of artificial intelligence," Commun. ACM, vol. 60, no. 10, pp. 27-31, Sep. 2017.

[23] M. Pitt, "Ways to avoid some common recruitment pitfalls," Hum. Resour. Manag. Int. Dig., vol. 17, no. 1, Jan. 2009.

[24] M. Prensky, "Digital natives, digital immigrants," On the Horizon, vol. 9, no. 5, pp. 1-6, 2001.

[25] PricewaterhouseCoopers, "Artificial Intelligence in HR: a No-brainer," 2017. [Online]. Available:

https://www.pwc.nl/nl/assets/documents/artificialintelligence-in-hr-a-no-brainer.pdf

[26] F. Rabiee, "Focus-group interview and data analysis," Proc. Nutr. Soc., vol. 63, no. 4, pp. 655-660, Nov. 2004.

[27] J. Ross, "The fundamental flaw in AI implementation," MIT Sloan Manag. Rev., vol. 29, no. 2, pp. 10-11, 2018.

[28] B. Ryan, "Applying technology in hiring," NHBR, vol. 40, no. 23, p. 12, 2018.

[29] M. Scherer, "AI in HR: Civil rights implications of employers' use of artificial intelligence and big data," SciTech Lawyer, vol. 13, no. 2, pp. 12-15, 2017.

[30] T. Tolan. "Why A.I. Will Never Replace Recruiters." hcinnovationgroup.com.

https://www.hcinnovationgroup.com/leadership-professionaldevelopment/blog/13030698/why-ai-will-never-replacerecruiters (accessed Sep. 28, 2020).

[31] A. K. Upadhyay and K. Khandelwal, "Applying artificial intelligence: implications for recruitment," Strategic HR Rev., vol. 17, no. 5, pp. 255-258, Oct. 2018.

[32] P. Van Esch, J. S. Black, and J. Ferolie, "Marketing AI recruitment: The next phase in job application and selection," Comput. Hum. Behav., vol. 90, pp. 215-222, Jan. 2019.

[33] D. Zielinski. "Recruiting Gets Smart Thanks to Artificial Intelligence." SHRM.org https://tinyurl.com/yypppycf 